\section{Linksseitige Lungenagenesie in Kombination mit linkspersistenter oberer Hohlvene und Cor triatriatum sinister bei einem 77-jährigen Mann}

Zusammenfassung: Es wird der Fall eines 77-jährigen Mannes berichtet, welcher im Endstadium seines kongenitalen Krankheitsbildes Aufnahme in unserem Krankenhaus fand. Nachdem im Rahmen einer rechtsseitigen Pneumonie die Respiratortherapie notwendig wurde, verstarb der Patient im weiteren Verlauf an einem chronischen Rechtsherzversagen.

Agenesia of the left lung in combination with a persisitent left superior vena cava and a cor triatriatum sinister in a 77 year olf man: We report the case of a 77 year old man, who was hospitalized in the final stadium of his congenital disease. Following a pneumonia in the right lung the respirator therapy was needed. Later the patient died by a chronic right heart failure.

\section{Falldarstellung}

Die Übernahme des bereits intubierten und beatmeten Patienten erfolgte aus einem Nachbarkrankenhaus zur Fortführung der Respirationstherapie. Dort hatte sich der 77jährige Mann wegen einer infektexazerbierten chronisch obstruktiven Bronchitis und Lungenemphysem in stationärer Behandlung befunden und im Verlauf eine $\mathrm{CO}_{2}$-Narkose entwickelt. Das „Fehlen einer Lunge“ sei bekannt gewesen. Bis auf rezidivierende bronchopulmonale Infekte in den letzten Jahren und eines stattgehabten linkshirnigen Insult waren keine Grunderkrankungen bekannt.

Befunde

77-jähriger intubierter und sedierter Patient in reduziertem Allgemein- und Ernährungszustand. Fehlendes Atemgeräusch über der linken Lunge, pneumonische Rasselgeräusche rechts basal. Cor nicht sicher auskultierbar. Herzfrequenz 130/min, Blutdruck: $50 / 30 \mathrm{~mm} \mathrm{Hg}$, Abdomen und Extremitäten unauffällig.

\section{Meier}

Innere Abteilung des St. Marien Hospitals Borken (Ltd. Abt. Arzt: Dr. med. H. Sartisson)

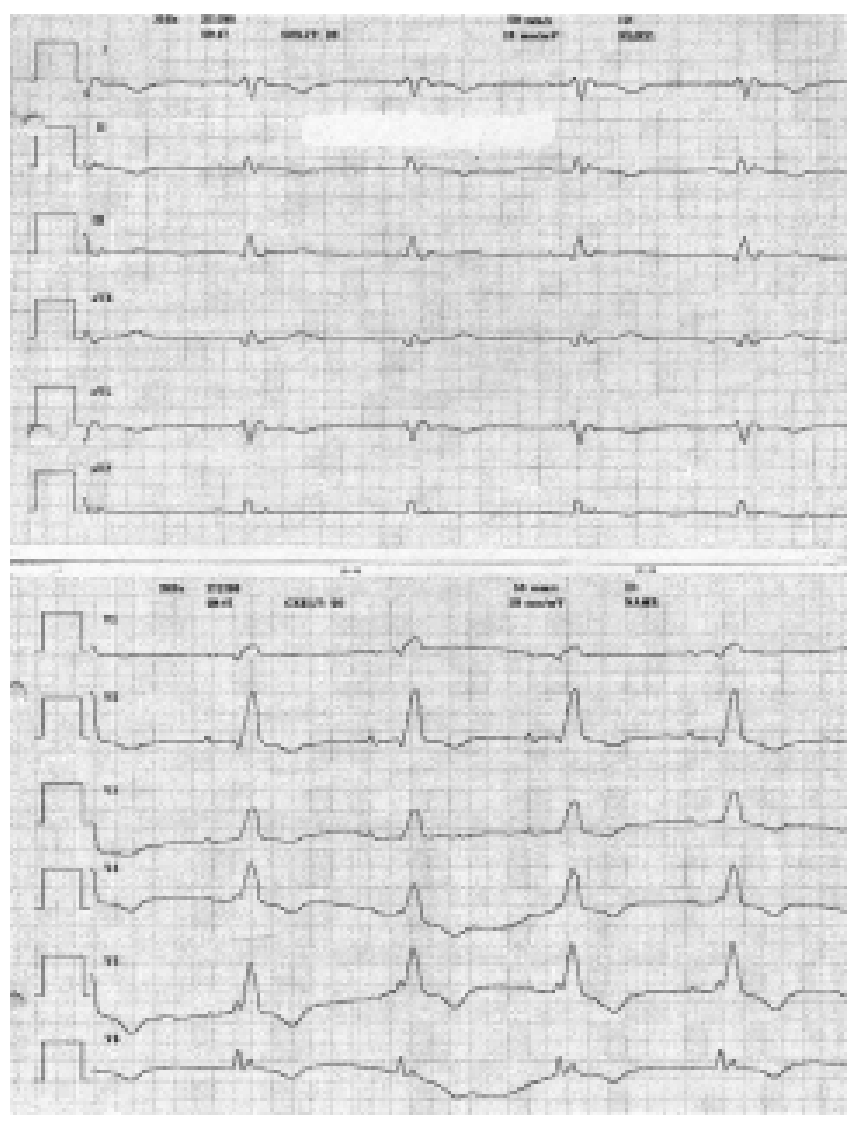

Abb. 1 12-Kanal-EKG.

Laborchemie

Blutgasanalyse $\left(\mathrm{FiO}_{2}=0,5\right): \mathrm{pH}: 7,44$

$\mathrm{pCO}_{2}: 46,0 \mathrm{~mm} \mathrm{Hg}$

$\mathrm{pO}_{2}: 89,1 \mathrm{~mm} \mathrm{Hg}$

$\mathrm{O}_{2}$ SAT: $96,7 \%$

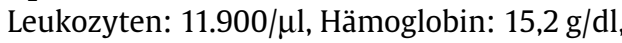

Hämatokrit: 48,7\%, MCV: $109 \mathrm{fl}$

GOT: 600 U/l, GPT: 849 U/l, Harnstoff: 95 mg/dl

Kreatinin: $1,35 \mathrm{mg} / \mathrm{dl}$ Lactat: $6,8 \mathrm{mmol} / \mathrm{l}$

EKG (Abb.1)

Rechtslagetyp, Sinusrhythmus, Rechtsschenkelblock in den linkspräcordialen Ableitungen.
Pneumologie 2000; 54: 249-251

(C) Georg Thieme Verlag Stuttgart · New York ISSN 0934-8387 


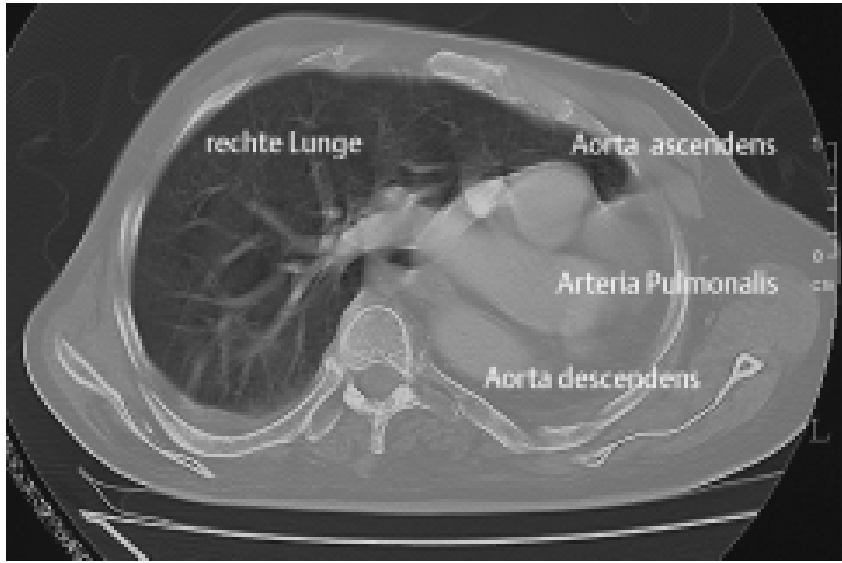

Abb. 2 CT-Thorax mit linksseitiger Lungenaplasie (in dankenswerter Weise überlassen von Dr. med. B. Kornmeier, Radiologische Abteilung des St. Marien Hospitals Borken $\mathrm{GmbH}$ )

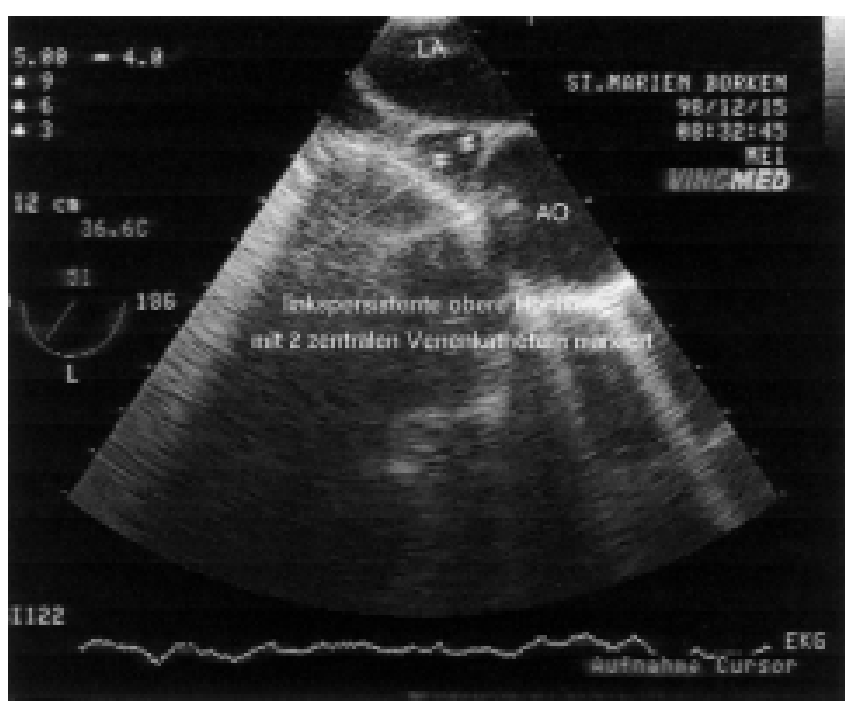

Abb. 3 TEE-transösophageal: linkspersistente obere Hohlvene $(\mathrm{LA}=$ linkes Atrium, $\mathrm{AO}=$ Aorta ascendens $)$.

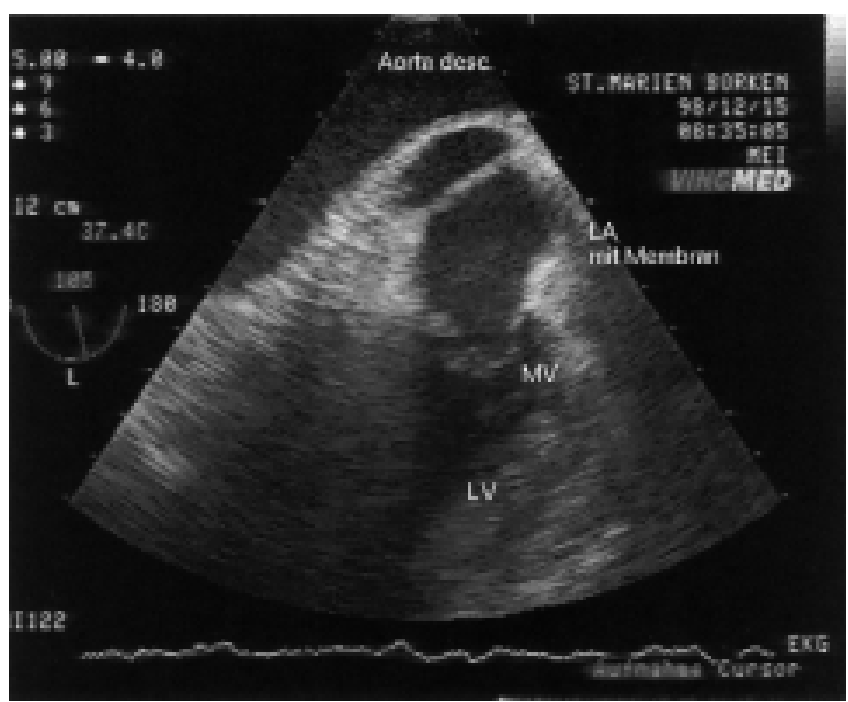

Abb. 4 TEE-transösophageal: 2-Kammer-Blick mit geteiltem linken Atrium ( $L A=$ linkes Atrium, $L V=$ linker Ventrikel MV = Mitralklappe).
Abdomen-Sonographie

Steatosis hepatis und Stauungsleber, Aortenatheromatose, Prostatahypertrophie

\section{Röntgen-Thorax}

Fehlen der linken Lunge mit Verziehung des Mediastinums nach links, Überblähung der rechten Lunge, Pneumonie rechts basal

\section{CT-Thorax (Abb. 2)}

Lungenagenesie links mit kompensatorischer Überblähung der rechten Lunge sowie Rotation des Herz-/Gefäßbandes in die linke Thoraxhälfte.

\section{Transösophageale Echokardiographie (Abb. 3 u. 4)}

Linksventrikuläre konzentrische Hypertrophie bei ungestörter linksventrikulärer Funktion. Ejektionsfraktion nach Teichholz 68\%. Rechtsventrikuläre konzentrische Hypertrophie und Dilatation. Geringe Trikuspidalklappeninsuffizienz. Cor triatriatum sinister bei völligem Fehlen der linksseitigen Vv. pulmonales. Fehlender linksseitiger Pulmonalarterienstamm. Linkspersistente obere Hohlvene.

\section{Diagnose}

Linksseitige Lungenaplasie in Kombination mit linkspersistenter oberer Hohlvene und Cor triatriatum sinister. (Aktuell: Respiratorische Insuffizienz und dekompensiertes Cor pulmonale chronicum mit ausgeprägter Stauungsleber bei rechtsseitiger Pneumonie und o.a. Grundkrankheit.)

\section{Verlauf}

Ursächlich für die respiratorische Insuffizienz mit initial septisch bedingtem Kreislaufversagen war eine pneumonische Mischinfektion mit Proteus mirabilis, Pseudomonas aeroginosa und diphteroiden Stäbchen. Nach kreislaufunterstützenden Basismaßnahmen wie Volumensubstitution und niedrig dosierter Katecholamingabe ließ sich das anfängliche Schockbild gut beherrschen. Eine gezielte antibiotische Therapie nach Antibiogramm erbrachte zwar eine rasche Verbesserung des Lokalbefundes, im weiteren Verlauf war der Patient jedoch nicht mehr vom Respirator zu entwöhnen und verstarb nach 3-wöchiger Intensivtherapie an dem präexistenten und jetzt dekompensierten Cor pulmonale chronicum.

\section{Diskussion}

Von der Hypoplasie der Lunge bis zur Agenesie oder Aplasie eines Lungenflügels und bis zum völligen Fehlen der gesamten Lungenanlage gibt es alle Übergänge. Derartige Missbildungen teilt Schneider [1] folgendermaßen ein:

a) einseitiges gänzliches Fehlen von Lunge und Bronchus

b) Fehlen einer Lungenhälfte und kurzer blinder Verlauf des zugehörigen Bronchus

c) Stammbronchus ausgebildet, Lunge als haselnuss- bis faustgroßes, ungelapptes, fleischiges Gebilde im Mediastinum gelegen 
d) geringere Grade der Hypoplasie mit Lungenrudiment im Pleuraraum

e) partielle Agenesie einer Lungenhälfte.

Die unter a) und b) genannten Fehlbildungen kommen sehr selten vor [2,3], etwas häufiger die übrigen [4]. Bei der Lungenagenesie werden oft, aber keineswegs immer Begleitmissbildungen beobachtet: Luftröhrenanomalien, Zwerchfelldefekt, Darmmissbildungen, Fehlen einer Niere, Gesichtsmissbildungen, Aurikularanhänge, angeborene Herzfehler und mehr. Zumeist handelt es sich um linksseitige Begleitmissbildungen [4-6]. Ein familiär gehäuftes Auftreten wird beschrieben, diesbezüglich ist die Familienanamnese hier jedoch unergiebig.

Bei unseren Patienten fand sich die Kombination mit einer linkspersistenten oberen Hohlvene und einem Cor triatriatum sinister. Obwohl das gemeinsame Auftreten einer linkspersistenten oberen Hohlvene mit einem Cor triatriatum sinister vereinzelt berichtet wird $[7,8]$, fand sich trotz intensivem Literaturstudium kein vergleichbarer dokumentierter Fall mit koexistentem Auftreten einer linksseitigen Lungenagenesie.

Sehr anschaulich sind die erhobenen Befunde des EKG mit rechtsschenkelblockartigen Veränderungen in den linkspräkordialen Ableitungen durch Rotation des Herz-Gefäßbandes. Die transösophageale Echokardiographie [9] beweist neben dem Befund des Cor triatriatum sinister die komplette Agenesie des linkspulmonalen Gefäßstammes im Rahmen der einseitigen Lungenagenesie.

Eine linkspersistente Vena cava superior mit Einmündung in den Koronarvenensinus (wie in unserem Fall), bereitet in den wenigsten Fällen hämodynamische Probleme. Anders gelagert ist die Situation bei Insertion in den linken Vorhof mit Rechtslinks-Shunt und hieraus resultierenden zusätzlichen Veränderungen in der Hämodynamik und Oxygenation [7].

Die hämodynamische Bedeutung des Cor triatriatum sinister und hieraus sich ergebende z.T. operative Korrekturnotwendigkeit ist wesentlich von dem Ausmaß des Durchtrittes durch die sehr variabel perforierten trennenden Membranen abhängig. Es werden Fälle ohne begleitende Obstruktion aber auch mit resultierender pulmonaler Hypertonie berichtet [10]. In fast allen Berichten stellt diese Fehlbildung jedoch ein arrhythmogenes Substrat vor allem auf Vorhofebene dar. Auch eine Rolle als Emboliequelle für rezidierende zerebrale und periphere Embolien (oder auch Lungenembolien beim Cor triatriatum dexter) wird diskutiert.

Eindrucksvoll ist in diesem Zusammenhang auch, dass diese komplexe kongenitale Herz-Lungenfehlbildung nicht mit der Einschränkung der normalen Lebenserwartung einhergehen muss. Bis auf den Beschwerdekomplex im Rahmen des Empyhsems der rechten Lunge und des damit verbundenen Cor pulmonale chronicum sowie des stattgehabten linkshirnigen Insultes (wohl kardioembolisch i.R. des Cor triatriatum sinister [10]) in den letzten Jahren konnte der 77 Jahre alt gewordene Patient auf ein vergleichsweise beschwerdearmes Leben zurückblicken.

\section{Literatur}

${ }^{1}$ Schneider P. Die Missbildungen der Atmungsorgane. In: Schwalbe E. Die Morphologie der Missbildungen des Menschen und der Tiere, Bd. III/2. Fischer Verlag Jena, 1912

${ }^{2}$ Danner D, Müller J, Steim H. Left lung aplasia with agenesia of the leftpulmonary artery. Radiologe 1969; 9: 83-85

${ }^{3}$ Haas RJ, Schäfer H, Sigmund E, Tosberg P. Unilateral pulmonary agenesia. Klin Pädiatr 1972; 184: 135-139

${ }^{4}$ Schröder J. Über das angeborene Fehlen einer Lunge. Zbl Allg Path 1958; 98: 555

${ }^{5}$ Lehmann W. Krankheiten und Missbildungen der Bronchien und der Lunge. In: Becker PE, Humangenetik. Thieme-Verlag, Stuttgart, 1972: 204-209

${ }^{6}$ Mirapeix RM, Domingo C, Sanudo JR, Mada JM. Unusual association of two unilateral anomalies present in adulthood: pulmonary hypoplasia and renal agenesis. Embryology and clinical expression. Surg Radiol Anat 1995; 17: 177-179

${ }^{7}$ Mazzuco A, Bortolotti U, Stellin G, Galluci V. Anomalies of the systemic venous return: a review. J Card Surg 1990; 5: 122-133

${ }^{8}$ Van Son JA, Hambsch J. Repair of subdivided left atrium associated with persistent left superior vena cava. J Thorac Cardiovasc Surg 1998; 116: 535

${ }^{9}$ Tantibhedhyangkul W, Godoy I, Karp R, Lang RM. Cor triatriatum in a 70-year-old woman: role of transesophageal echocardiography and dynamic threedimensional echocardiography in diagnostic assessment. J Am Soc Echocardiogr 1998; 11: 837-840

${ }^{10}$ Dauphin C, Lusson JR, Motreff P, Lorillard R, Briand F, Valy Y, Lamaisaon D, Chabrun A, Cassagnes J. Left intra-atrial membrane without pulmonary vein obstruction: benign condition of progressive evolution? Apropos of 7 cases. Arch Mal Coeur Vaiss 1998; 91: 615-621

Dr. med. M. Meier

Innere Abteilung St. Marien-Hospital GmbH

Am Boltenhof 7

46325 Borken 